\title{
DULOXETINA Y OTROS ANTIDEPRESIVOS TRICÍCLICOS: EFECTOS FARMACODINÁMICOS EN EL TRACTO URINARIO INFERIOR
}

\author{
R. VELA NAVARRETE, F.C. PÉREZ MARTÍNEZ, M. RAMÍREZ PÉREZ DEL YERRO, \\ J. CABRERA PÉREZ, C. GONZÁLEZ ENGUITA
}

Cátedra y Servicio de Urología. Fundación Jiménez Díaz. Universidad Autónoma de Madrid. Madrid.

Actas Urol Esp. 28 (3): 252, 2004

En relación con el artículo de revisión arriba mencionado, publicado en su revista en el número de noviembre/diciembre de 2003 (vol 27: 751-766), es oportuno hacer la siguiente consideración: Duloxetina no pertenece a la familia de los antidepresivos tricíclicos. Efectivamente, Duloxetina [(+)-N- metil-3-(1-naftaleniloxi)-2- tiofenepropanamina] encabeza ahora la lista de un nuevo grupo de fármacos antidepresivos, considerados inhibidores duales de la recaptación de serotonina y noradrenalina (SNRIs), junto con otros fármacos como la venlafaxina $\mathrm{y}$ milnacipram ${ }^{1-4}$. Duloxetina se une selectivamente y con una alta afinidad a los transportadores de serotonina y noradrenalina, y, a diferencia de los antidepresivos tricíclicos, no muestra afinidad significativa por receptores muscarínicos, histaminérgicos $\mathrm{H}_{1}, \alpha_{1}$-adrenérgicos, dopaminérgicos $\mathrm{D}_{2}$, opioides o serotoninérgicos $\left(5 \mathrm{HT}_{1 \mathrm{~A}}, 5\right.$ $\left.\mathrm{HT}_{1 \mathrm{~B}}, 5 \mathrm{HT}_{1 \mathrm{D}}, 5 \mathrm{HT}_{2 \mathrm{~A}}, 5 \mathrm{HT}_{2 \mathrm{C}}\right)^{5}$.

Los SNRIs como duloxetina, se diferencian de los antidepresivos tricíclicos por su falta de afinidad por otros receptores centrales y por su mejorada tolerancia y seguridad. Así, duloxetina no presenta los efectos anticolinérgicos o cardiovasculares evidenciados con los tricíclicos, ni presenta la toxicidad en sobredosis asociada a estos fármacos.

Es precisamente la falta de afinidad por estos receptores centrales propia de duloxetina y otros SNRIs lo que ha supuesto un avance muy importante en el campo de los antidepresivos dado que, manteniendo un alto nivel de eficacia, los SNRIs carecen de los efectos adversos que se asocian a los antidepresivos tricíclicos y que, por su mala tolerabilidad, impedian en muchos casos su utilización ${ }^{6}$. Ha sido precisamente, por su carácter antidepresivo que duloxetina haya sido incluida en algunos libros de texto farmacológicos junto a los antidepresivos tricíclicos tradicionales lo que ha motivado este equivoco y el incorrecto título de nuestra revisión, que no marca la diferencia debida entre este nuevo grupo de fárma- cos de acción dual y los tradicionales antidepresivos triciclicos; por otro lado, una familia bastante heterogénea, y algunos de ellos sin evidencias incontestables de efecto anticolinérgico.

Es esta una diferencia farmacológica que debe ser resaltada cuya evidencia científica puede encontrarse en publicaciones recientes ${ }^{7-10}$ en lo que se refiere a eficacia y seguridad comparativa, que será aún más evidente en publicaciones próximas antes de su llegada definitiva a la farmacopea española.

Hemos considerado que era importante resaltar estas singularidades de Duloxetina y la aparición de esta nueva familia de fármacos, para evitar equívocos como el señalado.

\section{REFERENCIAS}

1. ISHIGOOKA J.:Serotonin-noradrenaline reuptake inhibitors (SNRIs). Nippon Rinsho; 2001; 59(8): 1.523-1.529.

2. WONG DT, BYMASTER FP: Dual serotonin and noradrenaline uptake inhibitor class of antidepressants potential for greater efficacy or just hype? Prog Drug Res 2002; 58: 169-222.

3. TRAN PV, BYMASTER FP, MCNAMARA RK, POTTER WZ: Dual monoamine modulation for improved treatment of major depressive disorder. J Clin Psychopharmacol; 2003 Feb; 23 (1): 78-86.

4. BEIQUE JC, LAVOIE N, DE MONTIGNY C, DEBONNEL G : Affinities of venlafaxine and various reuptake inhibitors for the serotonin and norepinephrine transporters. Eur J Pharmacol 1998; 349 (1): 129-132.

5. LANTZ RJ, GILLESPIE TA, RASH TJ, KUO K, SKINNER M., KUAN HY, Knadler. MP: Metabolism, excretion, and pharmacokinetics of duloxetine in healthy human subjets. DMD , 2003; 31: 1.142-1.150.

6. LEONARD BE: New approaches to the treatment of depression. J Clin Psychiatry;: 1996; 57: 26-33.

7. BUMP R, HOOPER C, KOKE S, YALCIN I: Worldwide efficacy of duloxetine after 12 Weeks and 1 year in women with stress urinary incontinence (SUI): a 4-study meta-analysis. Neurourol Urodyn 2003; 22: 337.

8. YALCIN I, BUMP R: The effect of prior treatment experience and incontinence severity on the placebo response of Stress Urinary Incontinence (SUI). Neurourol Urodyn 2003; 22(5): 481.

9. DRUTZ H, CARDOZO L, BAYGANI S, BUMP R: Duloxetine treatment of women with only urodynamic stress incontinence awaiting continence surgery. Neurourol Urodyn 2003; 22(5): 523.

10. HURLEY D, et al. Duloxetine for stress urinary incontinence: a metaanalysis of safety. Neurourol Urodyn 2003; 22(5): 343. 